\title{
Scrutinize Study on Face Recognition by Pose Invariant Methodology
}

\author{
Deepika Dubey, G.S Tomar and Hye-jin Kim* \\ Business Administration Research Institute, \\ Sungshin W. University, \\ Bomun-ro 34da-gil, Seongbuk-gu, Seoul, Korea \\ deepika.sa1304@gmail.com,gstomar@ieee.org,hyejinaa@daum.net
}

\begin{abstract}
Three-dimensional (3D) face models can handle large pose face recognition problem. In this paper, we propose survey on pose-invariant face recognition method. By scrutinizing study depth, here we only analysis on pose invariant technique in face recognition method is able to handle self-occlusion and deformation, both of which are challenging problems in two-dimensional (2D) face recognition. Texture images of the face in the database can be the same view as the probe during surveillance. In this survey of face detection technology has been presented in this work feature based approach with low level analysis has been done. Information, color, shape, model etc. has been considered for investigation. The technique considered here also explained in other papers. Depth information has improved the performance of face recognition with large pose variations up to the mark able angle and under even more challenging conditions.
\end{abstract}

Keywords: face recognition, face detection, pose invariant, virtual frontal view, template matching, PCA, Three-dimensional (3D), Two-dimensional (2D), SVM, ANN, CNN, Edge detection.

\section{Introduction}

Face Recognition is an important research area in various field and disciplines nowadays. Face Recognition is a very simple terms are a process of recognition the face of a person and to identify the whole about that person. Face detection is the most studied topic in compute vision with the rapid increase in computational power. Many research project now a days working on face detection as well as on face recognition to demonstrate the capability for a computer to interact with human and to help humans in a natural way in which photos or snapshots are to be taken by the cameras.

Nowadays we are living in the $21^{\text {st }}$ century which is known for its technical and digital boom allowing the humans to develop the artificial intelligence. The digital innovations are providing the unconventional tools in the hands of millions of researchers to create the technology which can save the humans and the humanity as well as it can make us the digitally rich. In order to achieve the above mentioned task, we need to have a safe and secured environment which can only be achieved using the technology and developing that class of technological sensation.

The face recognition technology is the step towards the future in order to developing the secured environment. It is not at all about detecting the face to get an awesome snapshot with friends, it is all about replacing your every password entry with effortless face detection technology which can let $\mathrm{u}$ sit back and relax and you can access your credentials without entering anything in the system. It can also be used in the security purposes like if you have a database of all the wanted list in your region, $u$ can find them out from the crowd by using face detection of every individual and matching it with your database in real time process. [3] The advance development in the respective field makes 
the system powerful enough to detect the face without effecting the results by the effecting factors like age factor, weight factor, using beard on face, using external accessories on the face etc.

This technology have a very vast range of application in almost every field like it can be used in the forensic to recognize the details of dead body, it can also use by the banks to by allowing their customers to access their personal lockers by checking their authentication details by scanning their faces by which they can save so much time and energy, it can be used by the online payment gateways by replacing their conventional authentication technics by the whole new face detection and authentication process etc.

We are entering in the whole new era of technology and we have started being dependent on artificial intelligence either it is used for our comfort or our utility and in our security too. [1] We have more trust on technology, automation and artificial intelligence rather than the manual and human interference. In order to maintain its reliability, it must have to be more accurate and precise and it needs the continuous development and new innovation to achieve more and more precise results.

Nowadays our existing technology of face detection and recognition working well enough to detect the faces in very absurd conditions like the low light recognition, high contrast, more than the limit of background light, it can also detect the faces from different angles. But everything has a limit, it has a limit too. Beyond this limit it fails to achieve the desired results which can cause harm the reliability of this system. To expand its limit and in order to make it more precise, new research is needed which can help it to widen its boundaries of accessibility and make it more precise and accurate.

\section{Literature Survey}

In [6] face recognition is explored on the basis of feature extraction from range of images. The high level features are extracted from face to mark salient events in terms of lines, points and reasons on the face surface. The process has been under taken to provide segmentation of the data. The features generally considered are: nose bridge, nose base, nose ridge, eye corner cavities, inner outer convex center, eye socket boundary, convex center of eye, boundary surrounding nose etc. the considered face descriptors are diffused on the basis of relationship of depth and curvature values.

In [7] author have been proposed new algorithm for curve filtering, where is proposed that the new algorithms is capable of filtering automatically. The set of data points with geometrically. Cubic Bezier curves the algorithms is divided into two parts where significant points are identified in first step and curve fitting is done in second step. The algorithm has been applied to various digital images to check for the variety of proposal.

In [9] authors have analyzed posture on the basis of thumb rule. In this work four human posture have been considered by using K-G rule. The main component in these analysis is Eigen values, which is also named as Eigen features. The feature selection are statistical analysis was done on the basis of principle component analysis selection, where P- dimension features was replaced with much smaller M- dimensional feature space, and the spastically analysis was done on the basis of standard technique.. In this process ANN and SVM was used as classifier of the postures, which were under consideration on the basis of available images.

In [10] a survey of various face recognition techniques has been reported where in various methods have been investigated for their performance parameters in matching results. The neural network based training mechanisms have also been investigated for performance.

In [11] facial emotion recognition has been proposed in the back grounds of challenges faced while recognizing the faces during expression changes. In this method multi kernel SVM has been used detecting each action unit to calculate kernel matrix. Local Gabor binary pattern histogram is used and second kernel matrix has been computed from AAM coefficients RBF kernel. 
In [13] an attempt has been made to improve the performance of Eigen face method used for face recognition. In this method FAR and FRR have been composed for possible improvement. In this case the pre-processing model has been investigated and color normalization is taken up using intensity normalization concepts.

In [14] optimum image processing technique has been proposed identifying error rates of face recognition methods. It was also investigated that fisher face methods used for face recognition has not performed as predicted by the researchers. The image preprocessing has been used to improve the performance, however it has not been validated for certain data cases.

In [15] author have presented an approach of facial expression recognition using salient distance features. This approach is based on the feature obtained from patch based 2-D Gabor features, the performance has been analyzed on the basis of CRR (Correct Recognition Rate) with muscles movement also the approach has been used. State of the art techniques in the work.

\section{Sensitive Analysis}

After literature survey focus on some topic which is beneficial for the further research.

\section{a. Traditional Face Recognition Approach}

In traditional face recognition system just we have to learn the yearly survey In Photographic negation interferes with face recognition (Galper and Hochberg, 1971) they develop Pigmentation and shading is important in face recognition, after that The McGurk effect (McGurk and McDonald, 1976). I.e. the perceptual fusion of different lip-read and spoken syllables. This effect occurs even when the face is female and the sound male. Then Davies et al. (1978) compared recognition of monochrome Vs traced-drawings (no shading) in which Recognition of the drawings was very poor. Next in (1984) found that chins differences were detected first. After that Tanaka and Farah (1993) asked participants to learn faces. Then researchers find Facial expression/Face identificationBruce (1986) Young et al. (1986). Expression identified independently of identity.

Prosopagnosia can identify facial emotion. Some patients with dementia cannot identify facial emotion, but could identify famous faces.

Campbell et al. (1986) reported a severely prosopagnosia patient that still experienced the McGurk effect. Could also identify speech sounds from photographs. A second patient showed the reverse pattern.Hay and Young (1982) outlined stages of face recognition. Young et al. (1985) conducted a diary study. Most common errors:-

-A person was not recognized

-There was a feeling familiarity without identity

-A person was recognized but no name was retrieved

-A person was miss identified

\section{b. Face Recognition Based On Convolutional Neural Network (CNN)}

Face recognition still a challenging task now a days. The main challenge is that which algorithms is used to improve the performance of face recognition due to variability and effect that [3] included illumination variances, poses, facial expressions, occlusions, etc. face recognition is challenging task due to because of One factor is to varying poses of the facial image. The relative camera may either capture frontal, $45^{\circ}$, profile or upside down facial images. In this situation we used the algorithms which is based on neural network.

CNN is different because of unique architecture. It work on so many different task which is related to the image like segmentation, feature extraction, classification of detected face from the data base with preprocessing task on the given image. CNN tested on wide range of application related to face recognition approach. CNN applications are face detection, face recognition, gender recognition, object recognition, character 
recognition, texture recognition etc. $\mathrm{CNN}$ are of two type 4-layer and 2-layer $\mathrm{CNN}$ architectures for face recognition system. The first CNN architecture is designed for frontal images with occlusion, illumination variances and facial expressions. The second CNN architecture is designed for various poses, illumination variances and facial expressions.

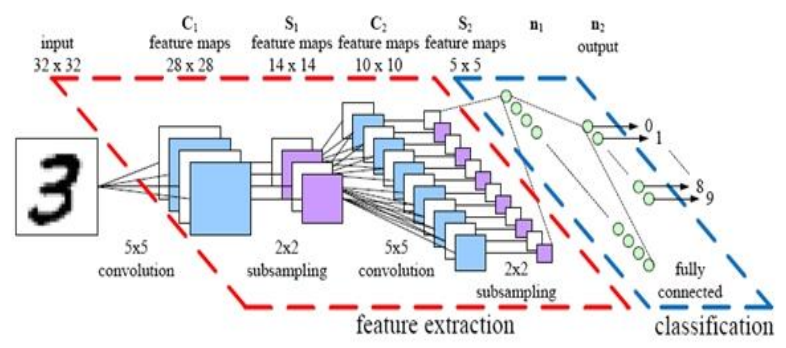

\section{Figure 1. Convolution Neural Network Architecture Model}

Convolution Neural Networks are very similar to ordinary Neural Networks they are made up of neurons that have learnable weights and biases. A Convolution Neural Network $(\mathrm{CNN})$ is next version of an ANN optimized for two-dimensional pattern recognition because it uses shared weights and less connections which greatly reduces the space.

\section{c. Gabor Based Face Recognition}

A Gabor filter, named after Dennis Gabor, is a linear filter used for edge detection. A face recognition system used in many application for correct authenticating a person from a digital image which is stored in database. Recognition is done on the basis of facial characteristics in the given image with the stored digital image database. Recognition are of two type face identification in which first we have to identify the faces from the picture or video and second is verification after identification verify with the given database and fetch whole information about that image. After identification and verification either to accept or to reject the person identity.

Face recognition basically depend upon two technique geometric technique and photometric technique. Geometric techniques included individual features, such as eyes, nose, mouth and head outline, and developing a face model based on position and size of these characteristics. Photometric approaches are statistical techniques that change or consider an image into values and compare these values with templates.

Gabor filter is related with the neural network technique. Gabor filter basically depend upon two things when it apply on the face recognition there mathematical terms and there biometric calculation. By using Gabor filter technique in face recognition getting revolutionary changes in this field.

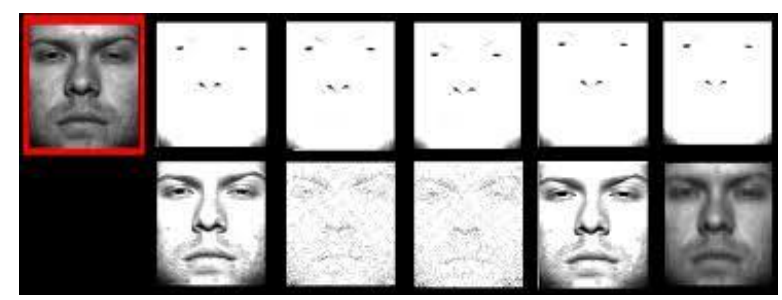

Figure 2. Gabor Based Face Recognition

Basically it is used for facial feature extraction from the input image and from stored database. 


\section{d. Model Based Face Recognition}

Face recognition basically done in two ways either from the static image or through video. Still images are easy to recognition and video is the collection of still images move in FPS (frame per second) so main advantages of video over still images is we are having multiple images or frames to provide better face recognition. In surveillance low resolution video are used containing so many faces and most of the faces are non frontal, poor contrast and having lighting condition problem. So in surveillance video it is very challenging task to find exact face recognition. To overcome this problem we use two model.

(a) 2D Active Appearance Models [Cootes and Taylor, 1998]

(b) 3D Morphable Models [Blanz and Vetter, 1999]

Basically these two approach at pose invariant technology 2D AAM and 3D Morphable model in face recognition system.

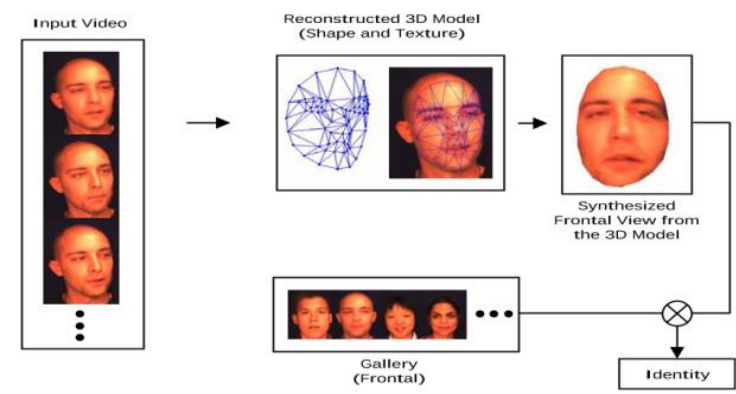

Figure 3. 3D Model-Based Face Recognition in Video [8]

3D head poses is in low resolution video is a challenging task because it is difficult to find continuous one-to-one mapping from person-identification low resolution to head pose parameters.

3D face models have been defining a way to merge for low resolution, poor contrast and non-frontal pose. We propose to overcome the pose problem by automatically (i) reconstructing a 3D face model from multiple non-frontal frames in a video, (ii) generating a frontal view from the derived 3D model, and (iii) using a commercial 2D face recognition engine to recognize the synthesized frontal view.

\section{e. Appearance based Face Recognition}

In appearance based face recognition, the features are selected by the pixel intensity value in the image of the face as an object. This pixel and its intensity directly propositional to the light emitted from the face in an image. That intensity value of the input image and intensity of these images are compared to the database images. Appearance based object recognition is more powerful than normal object recognitions.

Objects look different under varying conditions:

- $\quad$ Changes in lighting or color

- Changes in viewing direction

- $\quad$ Changes in size / shape

The Eigen light-field is used for set of features on base recognition, then recognize how the pixel intensities are used in appearance-based face and object recognition.

Appearance based approach based on 3 methods

- $\quad$ Directly correlation method

- $\quad$ Eigen face methods

- $\quad$ Fisher faces methods

There are too many factors which make face recognition difficult. Here basically we are discussion about angle of human faces taken by the camera or at the time of 
surveillance. There are generally six factors we need to concern: (1) illumination, (2) face pose, (3) face expression, (4) RST (rotation, scale, and translation) variation, (5) clutter background, and (6) occlusion. Appearance based models in which only the appearance is used, which are usually captured by different two-dimensional views of the object-ofinterest. Based on the applied features these methods can be sub-divided into two main classes, i.e. local and global approaches.

\section{f. Video Based Face Recognition}

Face recognition is now a day's recent topic in the field of image processing and Face recognition through videos is actively participate in research. As compare to static images, face recognition videos containing more information because video is the combination of number of the frames per second or we can say that video is the combination of so many number of the images. So if we need better result we required more options as a result in video face recognition we are getting better result. [15]

To improve the accuracy of face recognition in videos to get more robust and stable recognition will be achieved.

The video-based recognition has so more advantages over the image-based recognition. The information of faces can be utilized to the recognition task. Another benefit from video recognition is better effective representations, such as a 3D face model, resolution of the images improved it will be achieved by video move in FPS (frame per second). Video based recognition helps in learning or updating recognition for future frames. So if we concluded it video based face recognition is also a very challenging problem, which suffers from the given factors factor like low resolution quality of the facial images, scale variations, lightning condition, illumination changes, pose variations, Motion blur, occlusions and so many other things which affect the result at the time of detection or recognition of the faces.

There two other terms are use for matching of the images these terms are

FAR:-The False Acceptance Rate, is to calculate the biometric security system will incorrectly accept the wrong data during access of data for unauthorized user. A ratio of the number of false acceptances divided by the number of identification attempts.

FRR:-The False Rejection Rate is the probability that the system incorrectly rejects access to an authorized person, due to failing to match the biometric input with a template.

\section{Algorithms Analysis}

Algorithms analysis of face recognition technology is very important topic for the developer during their research. There is so many techniques which is to be covered during this study. Algorithm analysis is the initial basic step for develop new algorithms because without knowing the previous work we are not capable to find the new one. There is some limitation of the previous work we develop new techniques to improve the quality and for better result. 


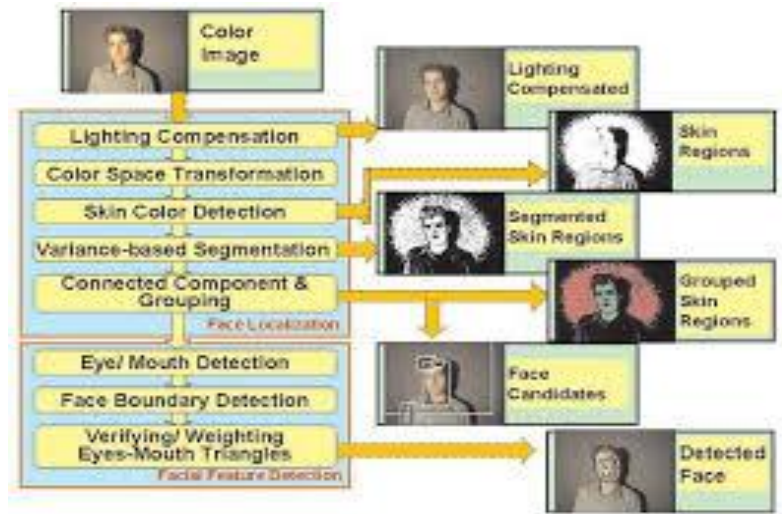

Figure 4. The Flowchart of the Face Detection Algorithm Analysis

\section{a. Eye mouth Detection}

Face recognition is recently used as biometric identification used in for personal identification, facial expression detection and so on. The features are extracted from the face and then it compair it to the stored database important face part such as eyebrows, eyes, noise, mouth are used to express expressionof the face.

- $\quad$ Active contour model

- $\quad$ Deformable template model

- $\quad$ Local smoothness of image density

- Color information of image

- Knowledge shape model

İn the above explained diagram number of step are used by implementing that steps we get better results from the given algorithms as previous results. Basically we focus on pose invarient in face recognition system in real time.

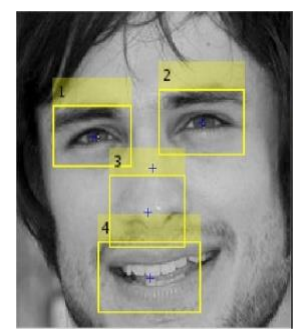

\section{Figure 5. Eyes and Mouth Detection}

We assume that the distance of right and left eyes is approximatelly equal to distance between the mouth and the middle of the eyes with an error of 10 pixels. İ this diagram left and right eyes mouth area as well as nose is also detected by square template. First face is to be detected andf after face detection facial feature is extracted for further processing.

\section{b. Face boundary with mark detection}

In face recognition system we have to extract facial feature by using algorithms as well we need to find whole face boundary which cover whole face as well as detect facial marks from face. Facial marks (e.g. moles, freckles, and scars) are also play a needful role in biometric recognition for uniquely identifying individuals. Facial marks play an important role in filtering a large database and distinguishing between ambiguous face comparisons such as identical twins. 


\section{c. Skin color/texture detection}

Skin detection is play an important role to find a correct face. Skin detection is the process of finding skin-colored pixels and regions in an image. This is a preprocessing step to find limbs /marks from human face. A skin detector transforms a given pixel into an appropriate color space and then use a skin classifier to label the pixel whether it is a skin or a non-skin pixel. Skin color and textures are important indication that people's health, age, wealth, beauty, etc. Skin detection means detecting image pixels from face and regions that contain skin-tone color.

\section{d. Lighting Conditions}

The [3] lightening conditions like background light, brightness, contrast, shadow etc. are not always same for the input. So the system must be smart enough to adjust these changes accordingly. Lightning conditions not only depend on the natural lights it will also depend on device camera quality as well as resolution of the image. These all condition effect on recognition of the face from the database.

\section{Future Research Direction}

This column helps the researcher who want to continue with this topic. In this future research directions are mentioned after detail survey on face recognition.

\section{a. Enlargement Of Face Recognition}

Face recognition system now a days work well and a recent topic used by researchers under different conditions, all face recognition system doing well job in frontal face snapshots and contrast lightning. All current face recognition algorithms fail under the varying conditions under which humans are able to identify other people by their [4] necked eyes and by use their memory. Next person recognition systems will need to recognize people in real-time and in much less time with different situations and getting maximum correct result.

Identification system that work in favorable environment, in the presence of noise and illumination changes, FAR and FRR vary. Cameras and microphones today are very small, light-weight and have been successfully integrated with wearable systems. Finally, researchers are beginning to demonstrate audio-and-video based person identification systems can achieve high recognition rates without requiring the user to be in highly controlled environments. But sometimes technology play a negative role during identification system. Like pose invariant, illumination, occlusion etc. so the recent research topic is depend on these topics.

\section{b. Transforming Images For Pose Invariant Face Recognition}

Face recognition has been attract attention of researchers due to its wide variety of applications, such as security, surveillance, law enforcement, and identity management. [9] With lot of lack in between these practical applications the face recognition task can be performed interfering, without user's awareness. Development of image sensing techniques helpful in it. During the past decades, performance of face recognition is improving but large pose variation problem still remains unsolved.

\section{i. 2D Transformation}

In past the developer work on [7] 2D based method explored the large pose face recognition problem and $2 \mathrm{D}$ based methods getting improvement. To achieve $2 \mathrm{D}$ detection deep learning is required learned face identity preserving features by using a 
deep neural network to transform a no frontal face to frontal view using millions of face images solve large pose variation problem using additional depth information.

\section{ii. 3D Transformation}

Passive 3D Based Method. 3D face models can be achieved from 2D face images. Here construct a 3D shape model from multiple 2D face images from the database and generated a set of sampled 2D face images as templates for pose-invariant recognition under frontal views. It can handle continuous pose variation up to \pm 45 in yaw and \pm 30 . Face recognition under large pose variation is still challenging. [7, 8]

Active 3D Based Method. With the development of active 3D sensing techniques, it is possible to capture 3D face models without considering the pose. There are two kinds of 3D sensors; one is depth cameras affordable and fast, but is of low resolution, low precision, and low reliability. The other is 3D scanners like producing high quality model but usually slow and expensive.

\section{Conclusion}

In this paper, we presented a pose-invariant face recognition method using different transformation methods and other pose invariant technologies using available face databases. According to survey the previous method cannot handle large pose variation problem, under challenge conditions like expression and occlusion. It also having disadvantage the proposed method is its high computational cost when identifying a person in the database. We are going to find better algorithms in above mentioned challenging conditions.

\section{References}

[1] L. Wiskott, J.-M. Fellous, N. Krüger, and C. von Der Malsburg, "Face recognition by elastic bunch graph matching," IEEE Transactions on Pattern Analysis and Machine Intelligence, vol. 19, no. 7, pp. 775-779, 1997.

[2] J. Wright, A. Y. Yang, A. Ganesh, S. S. Sastry, and Y. Ma, "Robust face recognition via sparse representation," IEEE Transactions on Pattern Analysis and Machine Intelligence, vol. 31, no. 2, pp. 210-227, 2009.

[3] X. Tan and B. Triggs, "Enhanced local texture feature sets for face recognition under difficult lighting conditions," IEEE Transactions on Image Processing, vol. 19, no. 6, pp. 1635-1650, 2010.

[4] Z. Zhu, P. Luo, X. Wang, and X. Tang, "Deep learning identity-preserving face space," in Proceedings of the IEEE International Conference on Computer Vision (ICCV '13), pp. 113-120, IEEE, Sydney, Australia, December 2013.

[5] Z. Zhu, P. Luo, X. Wang, and X. Tang, "Deep learning multi-view representation for face recognition," Technical eport, 2014, http://arxiv.org/abs/1406.6947.

[6] R. Jafri and H. R. Arabnia, "A survey of face recognition techniques," Journal of Information Processing Systems, vol. 5, no. 2, pp. 41-68, 2009.

[7] K. W. Bowyer, K. Chang, and P. Flynn, "A survey of approaches and challenges in 3D and multi-modal 3D+2D face recognition," Computer Vision and Image Understanding, vol. 101, no. 1, pp. 1-15, 2006.

[8] A. F. Abate, M. Nappi, D. Riccio, and G. Sabatino, "2D and 3D face recognition: a survey," Pattern Recognition Letters, vol. 28, no. 14, pp. 1885-1906, 2007.

[9] M. Ishimoto and Y.-W. Chen, "Pose-robust face recognition based on 3D shape reconstruction," in Proceedings of the 5th International Conference on Natural Computation (ICNC '09), pp. 40-43, Tianjin, China, August 2009.

[10] A. Asthana, T. K. Marks, M. J. Jones, K. H. Tieu, and M. V. Rohith, "Fully automatic pose-invariant face recognition via 3D pose normalization," in Proceedings of the IEEE International Conference on Computer Vision (ICCV '11), pp. 937-944, Barcelona, Spain, November 2011.

[11] B. Y. L. Li, A. S. Mian, W. Liu, and A. Krishna, "Using kinect for face recognition under varying poses, expressions, illumination and disguise," in Proceedings of the IEEE Workshop on Applications of Computer Vision (WACV '13), pp. 186-192, IEEE, Tampa, Fla, USA, January 2013.

[12] S. Gupta, K. R. Castleman, M. K. Markey, and A. C. Bovik, "Texas 3D face recognition database," inProceedings of the IEEE Southwest Symposium on Image Analysis \& Interpretation (SSIAI '10), pp. 97-100, IEEE, Austin, Tex, USA, May 2010. 
[13] C. Ciaccio, L. Wen, and G. Guo, "Face recognition robust to head pose changes based on the RGB-D sensor," in Proceedings of the 6th IEEE International Conference on Biometrics: Theory, Applications and Systems (BTAS '13), pp. 1-6, Arlington, Va, USA, October 2013.

[14] G.-S. J. Hsu, Y.-L. Liu, H.-C. Peng, and P.-X. Wu, "RGB-D-based face reconstruction and recognition,'IEEE Transactions on Information Forensics and Security, vol. 9, no. 12, pp. 2110-2118, 2014.

[15] K. I. Chang, K. W. Bowyer, and P. J. Flynn, "Multimodal 2d and 3d biometrics for face recognition," in Proceedings of the IEEE International Workshop on Analysis and Modeling of Faces and Gestures (AMFG '03), pp. 187-194, IEEE, Nice, France, October 2003.

[16] F. Hajati, A. A. Raie, and Y. Gao, "2.5 D face recognition using patch geodesic moments," Pattern Recognition, vol. 45, no. 3, pp. 969-982, 2012

[17] S. Gheisari, S. Javadi, and A. Kashaninya, "3D face recognition using patch geodesic derivative pattern, "International Journal of Smart Electrical Engineering, vol. 2, no. 3, pp. 127-132, 2013.

[18] O. L. Junior, D. Delgado, V. Gonçalves, and U. Nunes, "Trainable classifier-fusion schemes: an application to pedestrian detection," in Proceedings of the 12th International IEEE Conference on Intelligent Transportation Systems (ITSC '09), pp. 1-6, IEEE, St. Louis, Mo, USA, October 2009.

[19] B. Raducanu and F. Dornaika, "Pose-invariant face recognition in videos for human-machine interaction," in Computer Vision-ECCV 2012. Workshops and Demonstrations: Florence, Italy, October 7-13, 2012, Proceedings, Part II, vol. 7584 of Lecture Notes in Computer Science, pp. 566-575, Springer, Berlin, Germany, 2012.

[20] T. Shan, C. B. Lovell, and S. Chen, "Face recognition robust to head pose from one sample image," in Proceedings of the 18th International Conference on Pattern Recognition (ICPR '06), pp. 515-518, Hong Kong, China, August 2006. 